\title{
Novel Multiparametric Nomogram for Overall Survival Prediction in Complicated Intra-Abdominal Infection: A Multicenter Study in China
}

\author{
Sisi Huang ${ }^{1 \dagger}$, Limin Chen ${ }^{1+}$, Jiao Liu ${ }^{1,2}$, Sheng Zhang ${ }^{1}$, Lidi Zhang ${ }^{2}$, Zhenliang Wen ${ }^{2}$, \\ Yizhu Chen ${ }^{2}$ and Dechang Chen ${ }^{1,2 *}$ \\ 1 Department of Critical Care Medicine, Ruijin Hospital, Shanghai Jiao Tong University School of Medicine, Shanghai, China, \\ ${ }^{2}$ Department of Critical Care Medicine, Ruijin Hospital North, Shanghai Jiao Tong University School of Medicine, Shanghai, \\ China
}

\section{OPEN ACCESS}

Edited by:

Andrey V. Kozlov,

Institute for Experimental and Clinical

Traumatology (LBG), Austria

Reviewed by:

Anna Herminghaus,

Heinrich Heine University of

Düsseldorf, Germany

Longxiang Su,

Peking Union Medical College

Hospital (CAMS), China

*Correspondence:

Dechang Chen

chendechangsh@hotmail.com

†These authors have contributed equally to this work

Specialty section:

This article was submitted to Intensive Care Medicine and

Anesthesiology,

a section of the journal

Frontiers in Medicine

Received: 11 November 2020

Accepted: 29 January 2021

Published: 22 February 2021

Citation:

Huang SS, Chen LM, Liu J, Zhang S,

Zhang LD, Wen ZL, Chen YZ and

Chen DC (2021) Novel

Multiparametric Nomogram for Overall Survival Prediction in Complicated

Intra-Abdominal Infection: A

Multicenter Study in China.

Front. Med. 8:627416

doi: 10.3389/fmed.2021.627416
Background: Complicated intra-abdominal infections (clAls) in the abdominal cavity or within an abdominal organ are numerous and frequent dangerous entities in the treatment of critically ill patients. Early clinical evaluation is necessary.

Methods: This retrospective multicenter study included patients from 10 intensive care units (ICUs). Risk factors for the overall survival (OS) of patients with cIAl were selected using least absolute shrinkage and selection operator regression, and a nomogram was constructed subsequently. Calibration curve and receiver operating characteristic (ROC) curve were used to evaluate the calibration and discriminative ability.

Results: In total, 544 patients diagnosed with clAl were enrolled and divided into the study $(n=276)$ and validation $(n=268)$ sets. Sex, acute gastrointestinal injury, acute kidney injury, rare bacterium infection, Charlson score, and APACHE II score were identified as independent risk factors and were constructed for the nomogram. The nomogram showed marked calibration capability with a concordance index (C-index) of 0.909 and 0.831 in the study and validation set, respectively. Compared with the common clinical prognostic scoring system, the nomogram achieved the highest discrimination ability with an area under the curve (AUC) value of 0.91 and 0.83 in the study set and validation set, respectively.

Conclusions: Our newly constructed nomogram provides a useful tool for risk stratification and prognosis evaluation of clAl.

Keywords: nomogram, complicated intra-abdominal infection, prognosis, APACHE II, SOFA

\section{SUMMARY}

A multiparameter nomogram that especially included acute gastrointestinal injury (AGI) for complicated intra-abdominal infection (cIAI) prognosis evaluation was the first to be established. Sex, AGI, AKI, rare bacterium infection, Charlson score, and APACHE II score were identified as the risk factors. Compared with the commonly used scoring system in ICU, SOFA, and APACHE II, the nomogram presented better overall net benefits in decision curve analysis (DCA) and higher area under the curve (AUC) value in operating characteristic (ROC) curve. 


\section{INTRODUCTION}

Intra-abdominal infections (IAIs) are responsible for nearly $20 \%$ of sepsis cases and are the second most common cause of infectious morbidity and mortality after pneumonia in intensive care units (ICUs) (1). IAIs are further classified as uncomplicated and complicated. Complicated intra-abdominal infections (cIAI) are more likely to cause drug-resistant bacterium infections, surrounding organ damage, and even systemic inflammatory reactions, subsequently contributing to the accumulation of hospitalization costs, length of stay, and morbidity $(2,3)$. Mortality associated with cIAI is generally high at $23-38 \%$ (4, 5). Achieving prompt control over infection of an anatomic source is the cornerstone of cIAI management but is not always successful (4).

Several risk factors including delayed interventions, antibiotic-resistant pathogens, high severity of illness, advanced age, poor nutritional status, and pre-existing chronic medical conditions have been reported to cause treatment failure (6-9). Early clinical evaluation is essential for the illness stratification and the subsequent decision-making process, and even for auditing and research. However, specific scoring system is unavailable. Oddeke (10) also reported that "none of the widely-used scoring systems to predict overall outcome in critically ill patients are of clinical value." Nevertheless, few studies have comprehensively explored the risk factors for cIAI prognosis.

Therefore, this study aimed to identify factors that significantly influence the mortality of patients with cIAI in the ICU. Three different general organ function scores including the Charlson, Acute Physiologic and Chronic Health Evaluation II (APACHE II), and Sequential Organ Failure Assessment (SOFA) scores as well as several organ specific evaluation systems like the Glasgow Coma Scale (GCS), Chinese DIC scoring system (CDSS), acute kidney injury (AKI), and acute gastrointestinal injury (AGI) were investigated. We also incorporated patient characteristics, comorbidities, and infection source for a comprehensive assessment. The first nomogram for cIAI prognosis was constructed and confirmed in this study.

\section{METHODS}

\section{Study Population}

This was a retrospective, multicenter study conducted in 10 hospitals including Ruijin Hospital North, the First Affiliated Hospital of Wenzhou Medical School, The Second Affiliated Hospital of Zhejiang University School of Medicine, the First

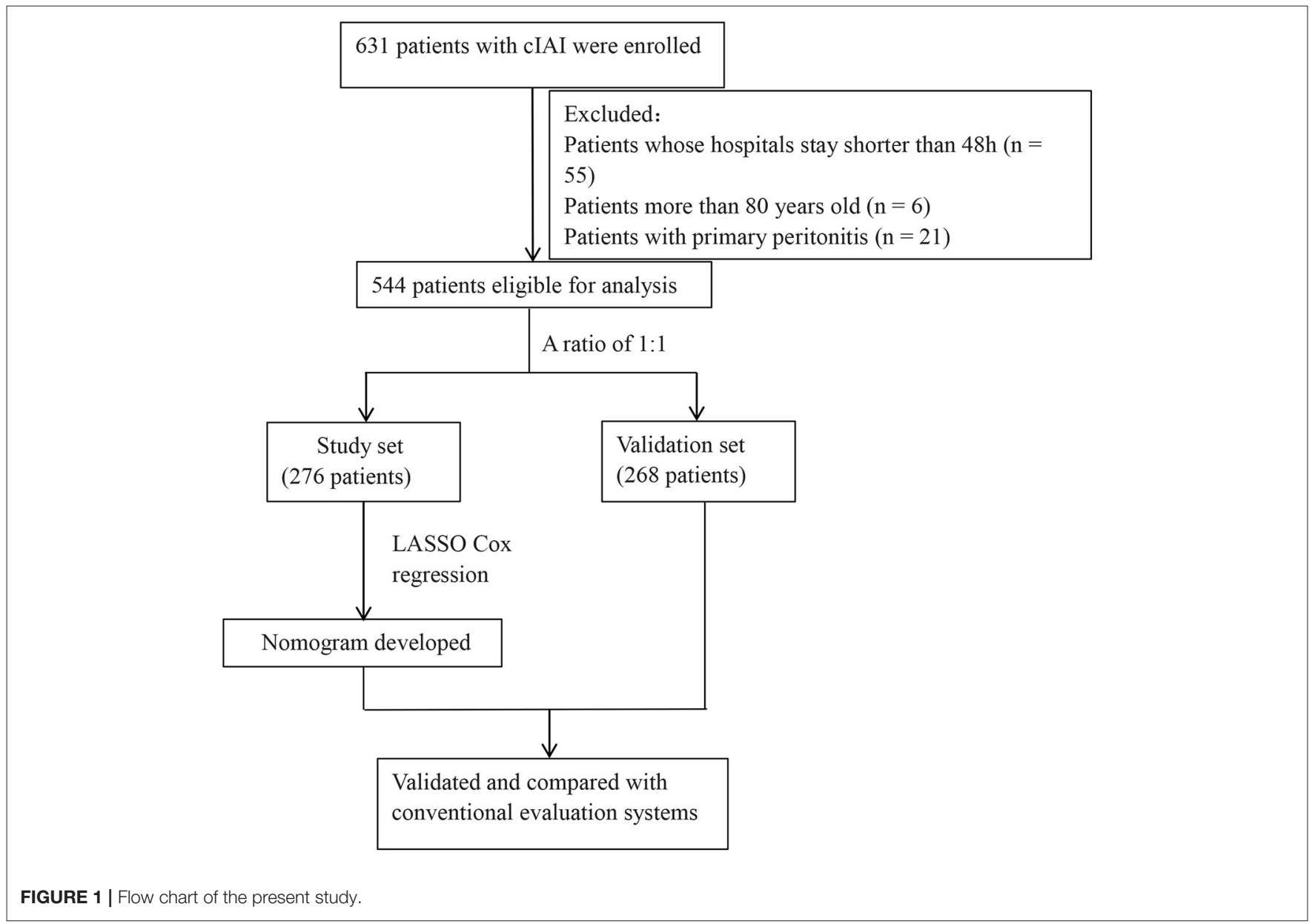


TABLE 1 | Demographic and clinical characteristics of study patients.

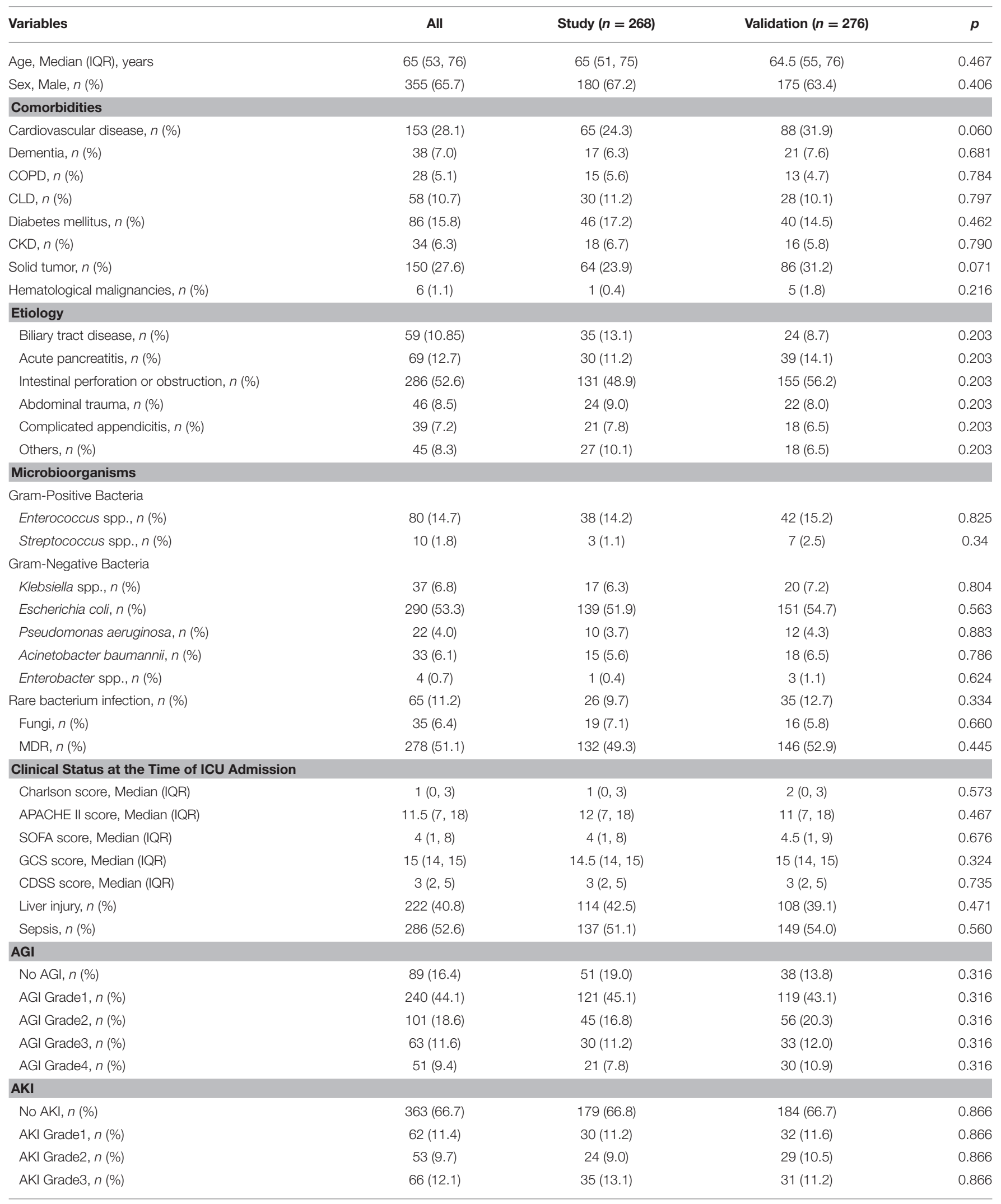


TABLE 1 | Continued

\begin{tabular}{lccc}
\hline Variables & All & Study $(\boldsymbol{n}=\mathbf{2 6 8})$ & Validation $(\boldsymbol{n}=\mathbf{2 7 6 )}$ \\
\hline Treatment variables & & $\boldsymbol{p}$ & \\
Glucocorticoid, $n(\%)$ & $94(17.3)$ & $42(15.7)$ & $52(18.8)$ \\
CRRT, $n$ (\%) & $67(12.3)$ & $33(12.3)$ & $34(12.3)$ \\
Inappropriate antibiotic exposure, $n(\%)$ & $27(5.0)$ & $10(3.7)$ & $17(6.2)$ \\
Outcomes & $18(11,32)$ & $18(11,30)$ & $18(12,34)$ \\
Length of hospitalization, Median (IQR), days & $9(4,16)$ & $9(4,17)$ & $8(4,16)$ \\
ICU duration, Median (IQR), days & $103(18.9)$ & $53(19.8)$ & 0.269 \\
In-hospital mortality, $n(\%)$ & $50(18.1)$ & 0.929 \\
\hline
\end{tabular}

APACHE II, Acute Physiologic and Chronic Health Evaluation II; SOFA, Sequential Organ Failure Assessment; GCS, Glasgow Coma Scale; CDSS, Chinese DIC scoring system; AGI,

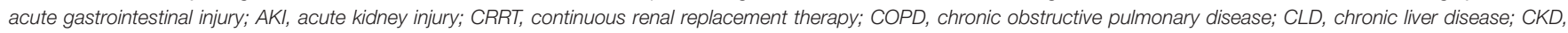
chronic kidney disease; MDR, multidrug resistance; ICU, intensive care units.

Hospital of Lanzhou University, the First People's Hospital of Kunshan, Huashan Hospital, Changhai Hospital, Minhang Hospital, Qingpu Branch of Zhongshan Hospital, and the Seventh People's Hospital of Shanghai University of Traditional Chinese Medicine from January 2017 to October 2018. In total, 544 patients (age, $18-80$ years) who were diagnosed with cIAI were enrolled in this study. Patients with primary peritonitis, with missing clinical data, or whose hospital stay was shorter than $48 \mathrm{~h}$ were excluded. This retrospective study was reviewed and approved by the Ruijin Hospital North. The included patients were randomly divided into a study set $(n=276)$ and validation set $(n=268)$ at a ratio of $1: 1$.

\section{Date Collection}

The patient characteristics and clinical data of each patient were carefully collected and scrutinized. Clinical data such as the Charlson score, APACHE II score, SOFA score, GCS score, DIC score, AGI Grade, AKI Grade, and liver function were acquired on the first day in the ICU. Variables related to intra-abdominal infection included infection sites such as the biliary system, pancreas, and intestine, abdominal trauma, spontaneous peritonitis, and others. Pathogens having the highest drug resistance during hospitalization were recorded. Pathogens were classified into Gram-positive bacteria (Enterococcus spp., Streptococcus spp.), Gram-negative bacteria (Escherichia coli, Klebsiella, Acinetobacter baumannii, Pseudomonas aeruginosa, and Enterobacter spp.), rare bacterium infection (see Definitions), and fungi. Comorbidities occurring before admission included stroke, chronic obstructive pulmonary disease (COPD), chronic liver disease (CLD), diabetes, chronic kidney disease (CKD), malignancy, and hemopathy. The day of discharge or death was considered as the end point of the study.

\section{Definitions}

Complicated intra-abdominal infection (cIAI) was defined as a generalized inflammatory process extending beyond the hollow viscus of origin into the peritoneal cavity that affects multiple organs and causes abscesses or peritonitis (11). Rare bacterium infection was defined as seldom-seen bacterium infection of cIAI such as Proteus spp., Serratia spp., Staphylococcus spp., and Stenotrophomonas maltophilia. Multidrug-resistant (MDR) bacteria refer to the bacteria that are resistant to three or more kind of commonly used antibiotics, including extensive drug resistance $(\mathrm{XDR})$ and pan-drug resistance (PDR).

\section{Construction and Validation of the Nomogram}

We incorporated all the clinical data as prognostic features to select the most useful predictive variables in the study group. The least absolute shrinkage and selection operator (LASSO) regression with 10 -fold cross-validation was used to shrink all the regression coefficients toward zero. The penalty parameter lambda controls the amount of shrinkage, so lambda. min [the Lambda at which the minimal MSE (Mean Square Error) is achieved] was identified at first, and lambda. 1sd (one standard deviation of lambda. min) was used to select features for the nomogram construction of cIAI overall survival (OS).

A calibration curve was used to assess consistency between the nomogram-predicted survival probability and the actual fraction survival probability. According to the median risk probability of death predicted by the nomogram, patients with cIAI were classified into high- and low-risk groups. The potential association of the nomogram score with OS was first assessed in the study cohort and was then validated in the validation cohorts using Kaplan-Meier survival analysis. The clinical utility of the nomogram model was assessed by a decision curve analysis (DCA) in the testing and independent validation cohorts by quantifying the net benefits at different threshold probabilities. The receiver operating characteristic (ROC) curve and area under the curve (AUC), which is useful to estimate the predictive accuracy of prognostic predictors, were also used to assess and compare the performance of the nomogram and conventional evaluation systems such as APACHE II score, and SOFA score. A larger AUC indicated more accurate prognostic stratification.

\section{Statistical Analysis}

Continuous variables not following normal distribution were expressed as median [interquartile range (IQR)] and analyzed using the rank-sum test. Categorical variables were expressed 
as frequency or ratio and were analyzed using the $\chi^{2}$ test. All statistical analyses were performed using $\mathrm{R}$ (version 3.6.2). The "glmnet" package was used to perform the LASSO Cox regression model analysis. All statistical tests were two-sided, and $P$-values $<0.05$ were considered statistically significant.
RESULTS

\section{Clinical Characteristics}

Of 631 patients who was confirmed with cIAI, 544 patients were eligible for analysis (Figure 1). The clinical characteristics of the

A

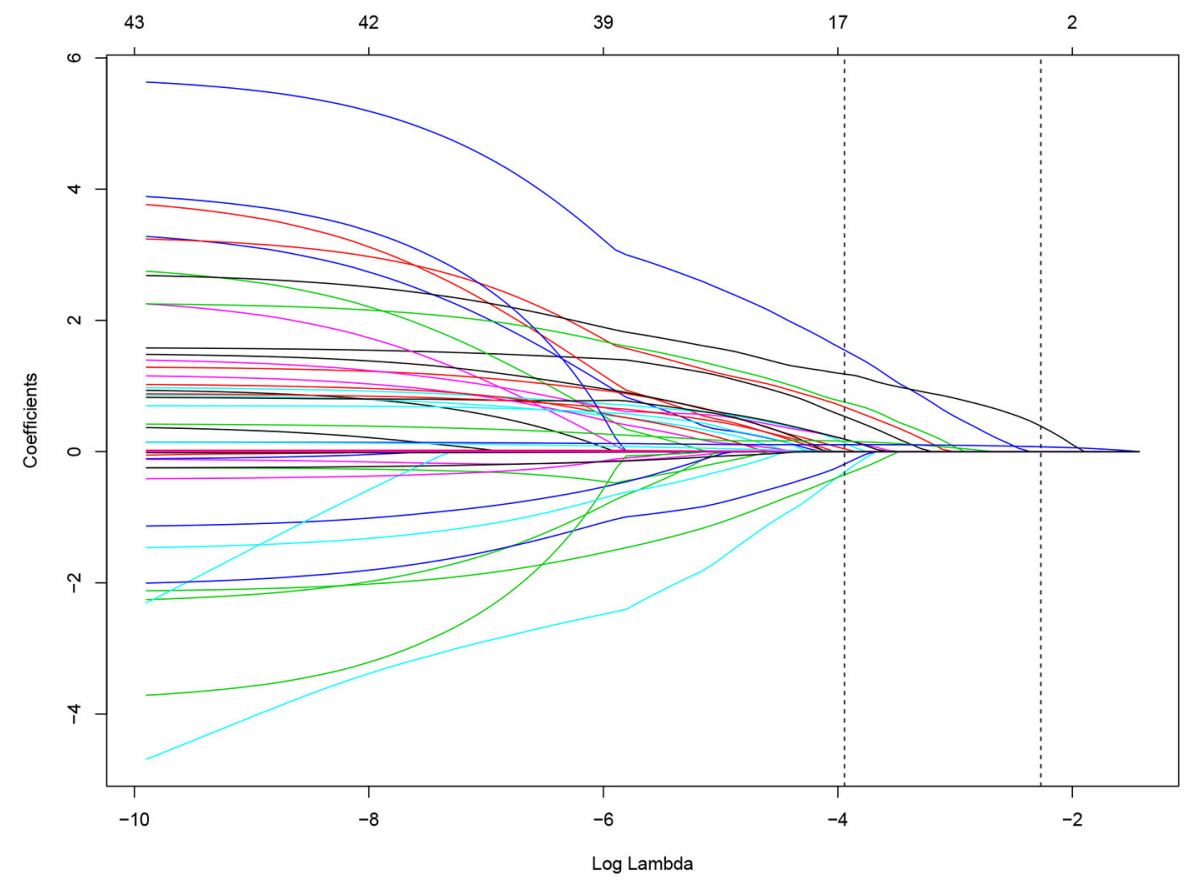

B

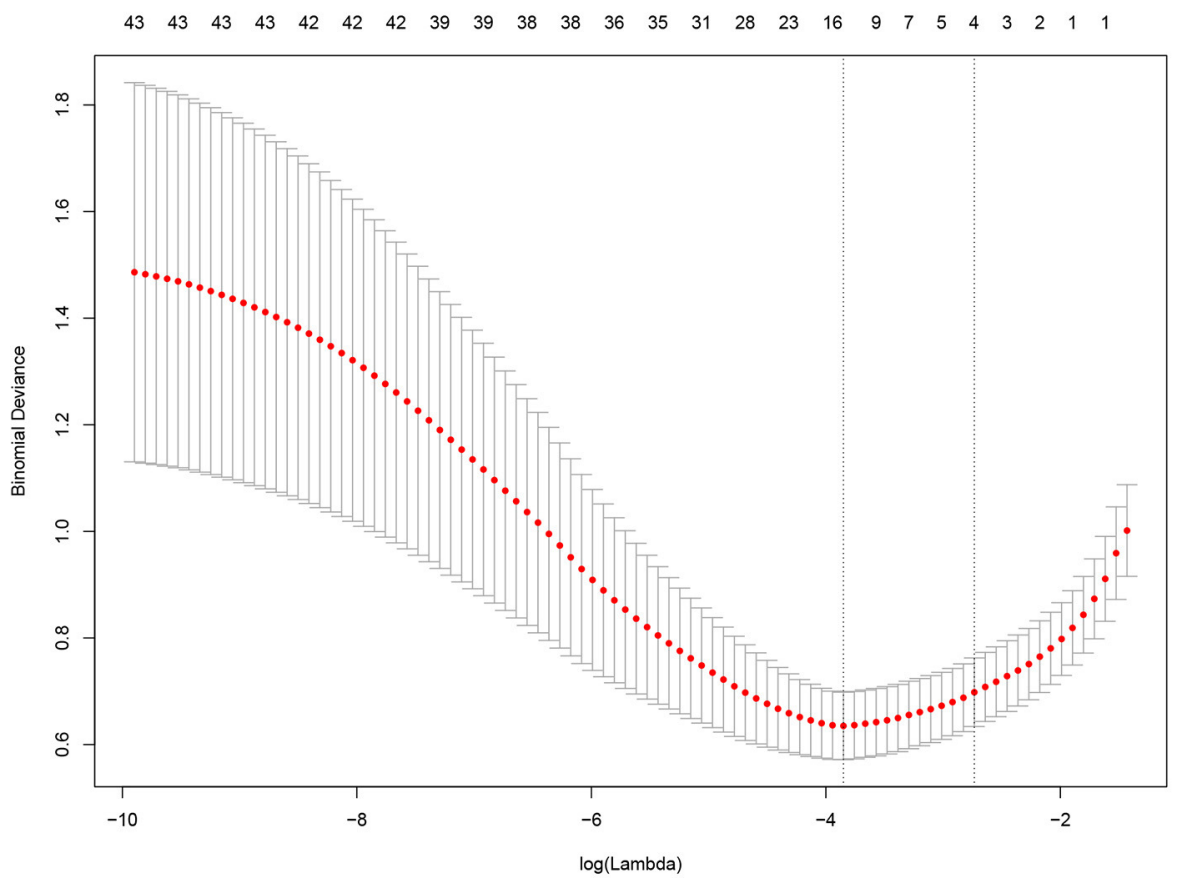

FIGURE 2 | Risk factor selection using the least absolute shrinkage and selection operator (LASSO) Cox regression model. (A) LASSO coefficient profiles of the 35 factors for OS. (B) Tuning parameter (lamda) selection in the LASSO model used 10-fold cross-validation via minimum criteria for OS. 
study and validation cohorts are shown in Table 1 . The study and validation groups included 268 and 276 patients, respectively. There were 137 (51\%) patients in the study set and 149 (54\%) patients in the validation set that also had sepsis. The hospital duration of the study set was 18 (IQR, 11-30) days and the ICU duration was 9 (IQR, 4-17) days. The hospital duration of validation set was 18 (IQR, 12-34) days and the ICU duration was 8 (IQR, 4-16) days. The mortality in the study and validation set was $20 \%$ and $18 \%$, respectively. All characteristics were wellbalanced in both the study and validation cohorts $(p>0.05)$.

\section{Nomogram Development}

A LASSO Cox regression model was used as a prognostic classifier, which successfully identified six potential predictors from the 37 features with non-zero coefficients in the study cohort (Figure 2). Sex, AGI, AKI, rare bacterium infection, Charlson score, and APACHE II score were independent risk factors (Figures 2, 3). A nomogram was constructed subsequently (Figure 3).

\section{Validation of Nomogram}

The nomogram was well-calibrated as revealed by the calibration curves, and its prediction of death showed a good correlation between the actual observed outcome and the nomogram prediction (Figures $4 \mathbf{A}, \mathbf{B})$ in the study group $(p>0.05)$. This was further verified in the validation cohort $(p>0.05)$. The C-index of the nomogram for the prediction was 0.909 and 0.831 in the study and validation set, respectively. Accordingly, patients were classified into low-risk and high-risk groups based on the nomogram. Kaplan-Meier survival analysis showed that the actual survival rate of cIAI differed significantly from patients with low risk to those with high risk in both the study and validation sets $(p<0.001)$ (Figures 4C,D).

\section{Comparison of the Nomogram With Conventional Evaluation Systems}

The DCA in the study cohort showed that our multiparametric nomogram had a better overall net benefit compared to the SOFA Score, APACHE II Score, and the treat-all patients strategy or the treat-none strategy at different threshold probabilities across the majority of the range between 4 and 100\% (Figure 5A). DCA in the validation cohort showed an equal net benefit with the nomogram and the SOFA Score or APACHE II Score (Figure 5B).

The ROC curves (Figures 5C,D) were used to assess the discrimination ability of nomogram, SOFA, and APACHE II for the mortality of IAI. AUC values were the highest for the nomogram in the study group $(\mathrm{AUC}=0.91)$ (Figure $5 \mathrm{C})$, and the validation group $(\mathrm{AUC}=0.83)$ (Figure 5D). The performances of SOFA and APACHE II are summarized in Table 2.

\section{DISCUSSION}

We conducted a multicenter retrospective study in which we successfully enrolled 544 patients with cIAI to construct a nomogram for the evaluation of mortality risk. Sex, AGI, AKI, rare bacterium infection, Charlson score, and APACHE II score were identified as the risk factors, and were used to constitute the nomogram for prognosis prediction of cIAI in the study cohort. Internal validations further confirmed the nomogram as a successful prognostic evaluation system. Compared with the commonly used scoring system in ICU, SOFA, and APACHE II, the nomogram presented better overall net benefits in DCA and higher AUC value in ROC, which demonstrated the incremental value for evaluation of cIAI prognosis.

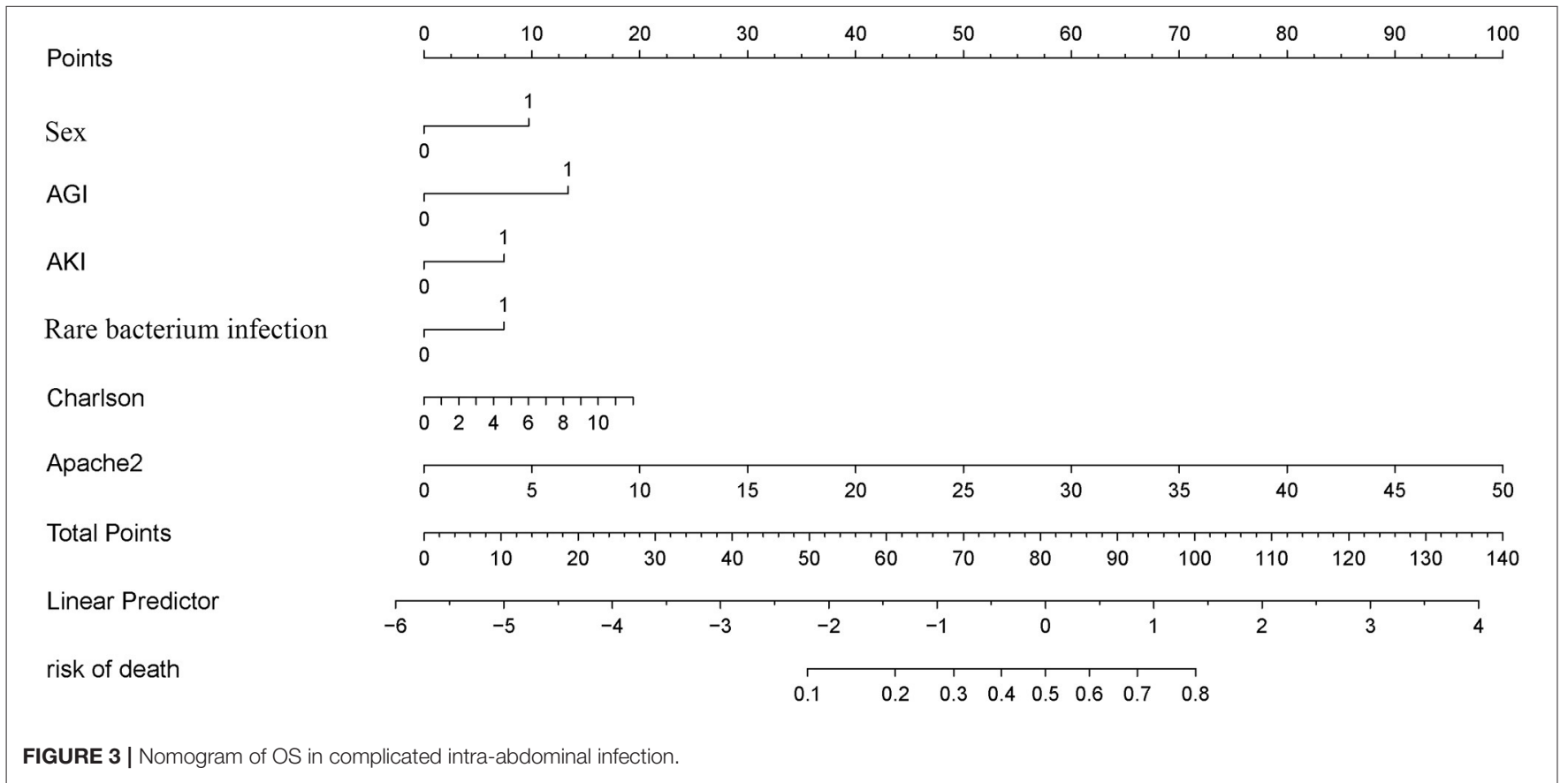




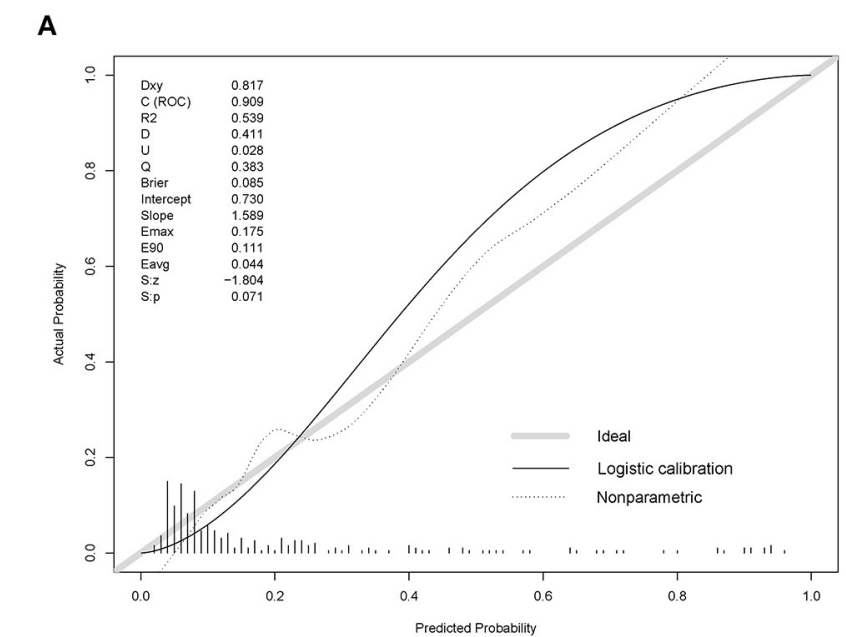

C
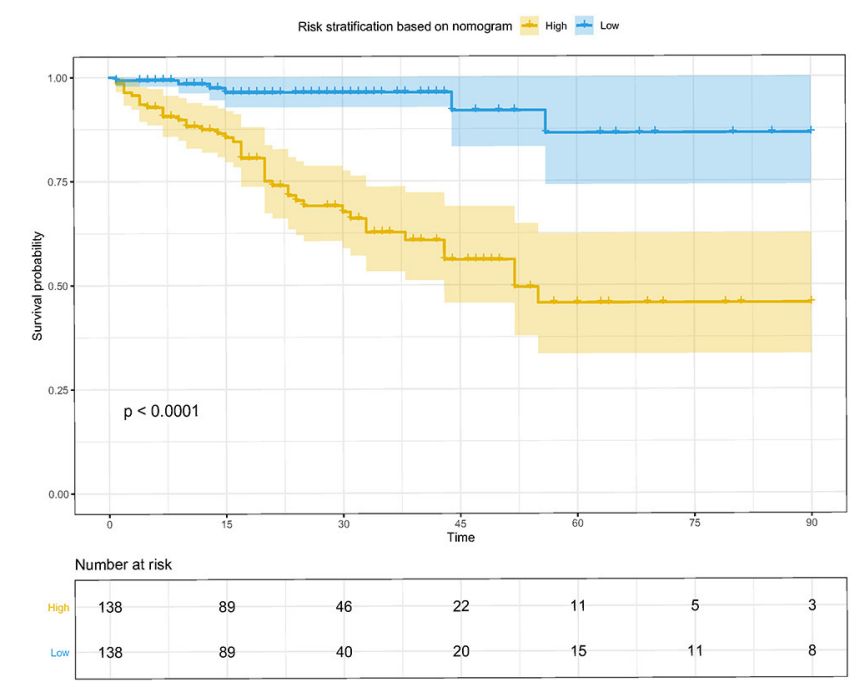

B

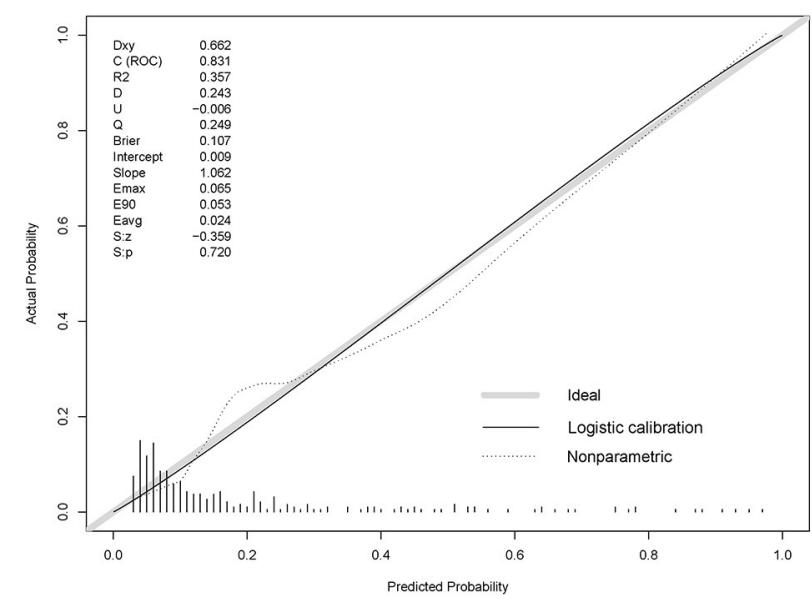

D

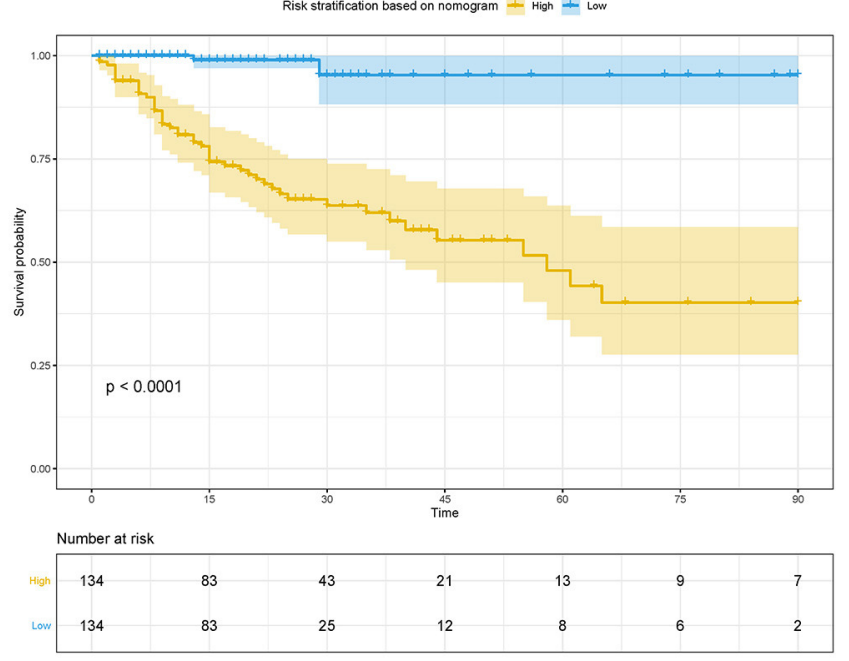

FIGURE 4 | Calibration curves and Kaplan-Meier survival curves for the nomogram in study cohort and validation cohort of clAl. (A) Calibration curve of the nomogram in the study cohort. (B) Calibration curve of the nomogram in validation cohort. (C) Kaplan-Meier survival curve of the nomogram in the study cohort. (D) Kaplan-Meier survival curve of the nomogram in the validation cohort.

The Complicated Intra-Abdominal infection Observational Worldwide (CIAOW) study designed by the World Society of Emergency Surgery (WSES) had identified a critical clinical condition (severe sepsis and septic shock) upon hospital admission and was the most significant risk factor for death of cIAI (12). SOFA and APACHE II scores are commonly used in ICU for severity evaluation and are evaluated for the severity of cIAI (13-16). However, the prognosis prediction ability of APACHE II or SOFA score was controversial. Pascal et al. reported that APACHE II score was associated with the presence of Pseudomonas aeruginosa in peritoneal fluid culture but not with the prognosis (17). In a study of Kulkarni et al., APACHEII score between 11 and 20 but not APACHE-II scores of 1 to 10 or $>20$ was shown to be a predictor of risk of mortality in patients with peritonitis due to hollow viscus perforation (18). Another study identified APACHE II $\geq 13$ as the independent risk factors for failure of initial antibiotic therapy of cIAI (19). We identified APACHE II score but not SOFA score as the risk factor for patients with cIAI. According to our newly constructed nomogram, APACHE-II score between 11 and 20 achieved a point of 20 to 40 and APACHE II $\geq 13$ achieved a point $\geq 26$. The APACHE II score in the whole cohort ranged from 1 to 49 with a point range from 2 to 98 (Figure 3). We further compared the prognosis prediction ability of our newly constructed nomogram with APACHE II, SOFA score. Both DCA and ROC curves identified a better overall net benefit and better discrimination ability of the nomogram compared with APACHE II, SOFA score in the study group, even though the 

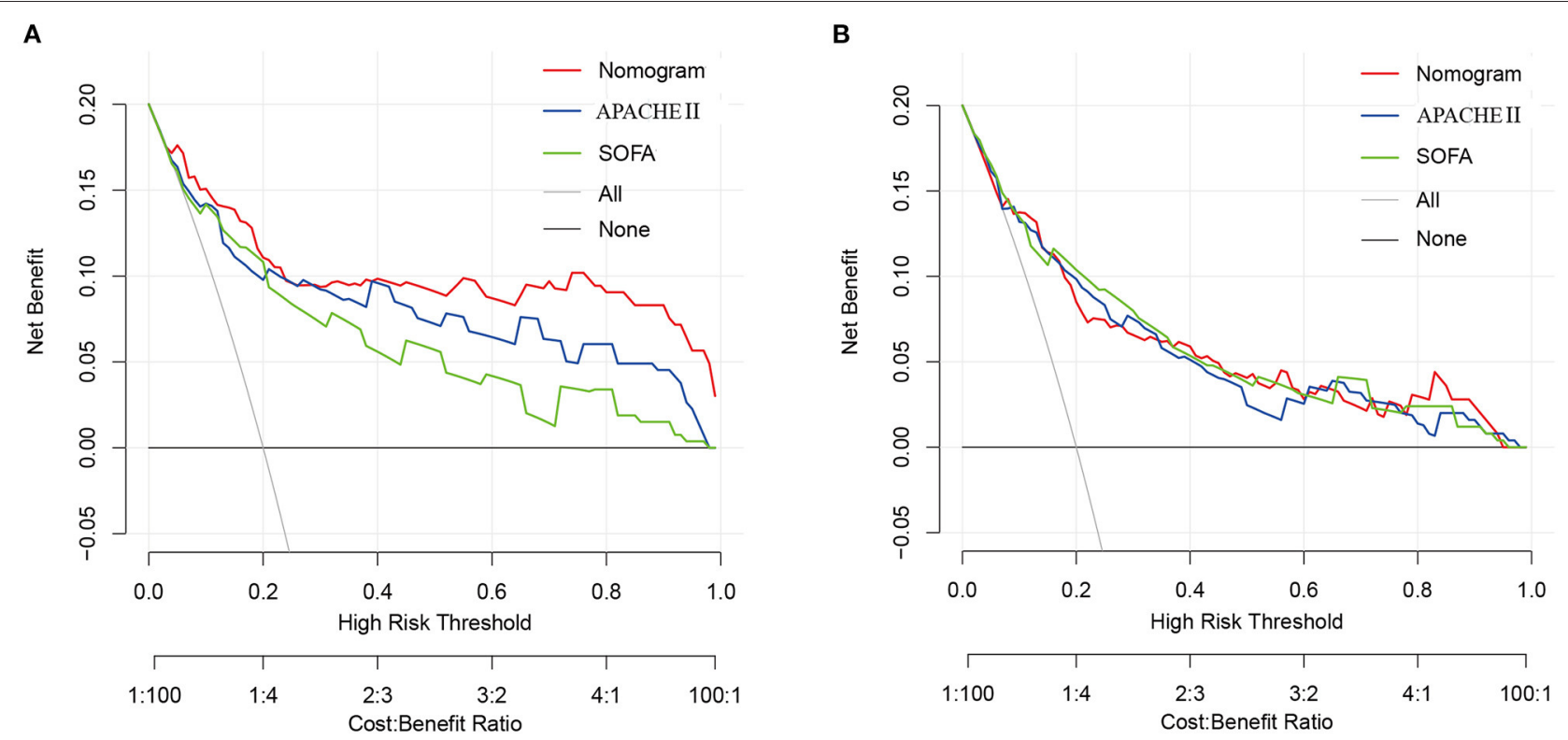

C

\section{D}
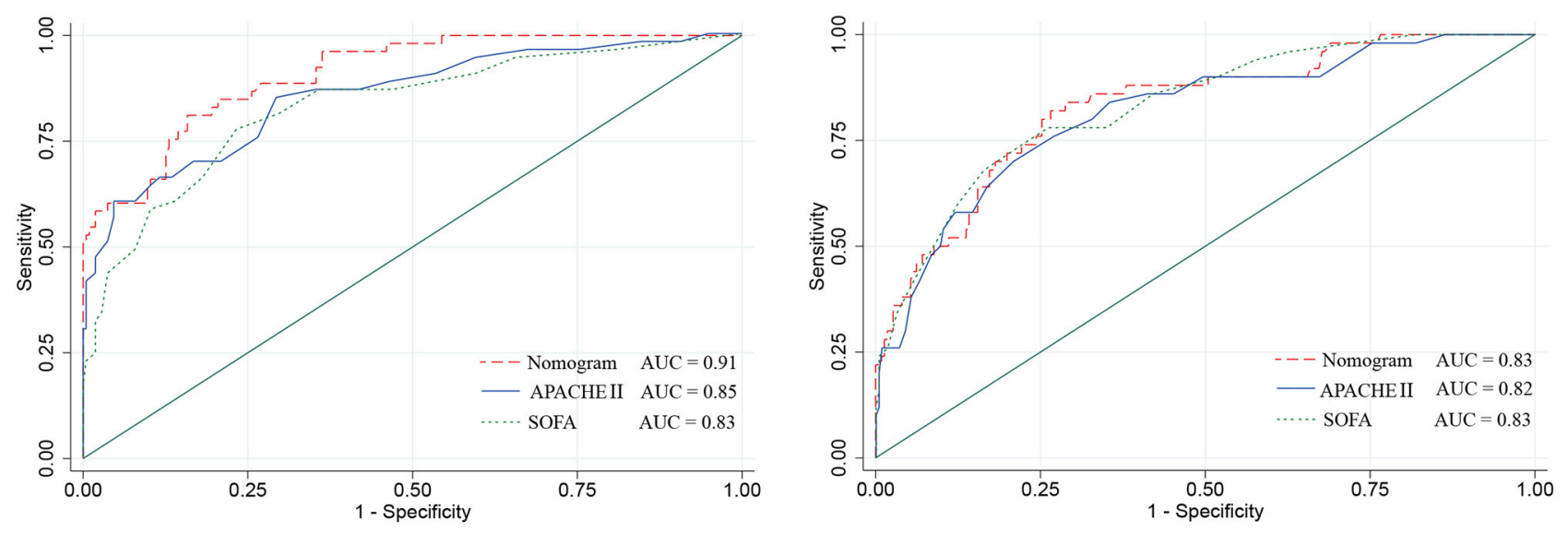

FIGURE 5 | Decision curve and ROC curve for the nomogram in the study cohort and validation cohort of cIAl. (A) Decision curve of the nomogram in the study cohort. (B) Decision curve of the nomogram in the validation cohort. (C) ROC curve of the nomogram in the study cohort. (D) ROC curve of the nomogram in the validation cohort.

comparation of the three in the validation cohort showed an equal benefit.

Effects on function of specific organs, especially the gastrointestinal system, which was first and foremost affected, should be considered. The Working Group on Abdominal Problems of the European Society of Intensive Care Medicine firstly developed the definitions of AGI with four grades of severity, making it possible to estimate the gastrointestinal function of critically ill patients (20). AGI is widely used in ICU $(21,22)$. A multi-prospective study that recruited patients admitted to ICU diagnosed with AGI showed that AGI grading was positively correlated with all-cause mortality $(23,24)$. A retrospective study enrolled 286 critically ill patients with acute pancreatitis from ICU; the AGI grade distribution was $34.62 \%$ with grade 1, 22.03\% with grade 2, $32.52 \%$ with grade 3 , and $10.84 \%$ with grade 4 , and the AGI grade was identified useful for predicting mortality (AUC $=0.854$ ) (25). AGI grade upon ICU admission was firstly investigated for patients with cIAI in this study and was indicated as a risk factor of death. Patients with AGI got a point of 13 according to the nomogram (Figure 3). AKI is a common disease in the critically ill individuals, and is associated with high mortality (26). Alejandro's study showed that the incidence of AKI in surgical septic patients with secondary peritonitis was $58.8 \%$ (15). This study also had 181 (33.3\%) patients with cIAI developed into AKI (Table 1) and identified 
TABLE 2 | AUC for Nomogram, APACHEll, and SOFA in the study cohort and validation cohort.

\begin{tabular}{|c|c|c|c|c|c|c|c|}
\hline Predictors & AUC & $95 \% \mathrm{Cl}$ & $\begin{array}{c}\text { Sensitivity } \\
(\%)\end{array}$ & $\begin{array}{c}\text { Specificity } \\
(\%)\end{array}$ & $\begin{array}{l}\text { Correctly } \\
\text { classified }\end{array}$ & PPV & NPV \\
\hline \multicolumn{8}{|c|}{ Study cohort } \\
\hline Nomogram & 0.91 & $0.22-5.13$ & 81.13 & 84.19 & $83.58 \%$ & $80.00 \%$ & $90.79 \%$ \\
\hline APACHE ॥ & 0.85 & $0.21-2.90$ & 84.91 & 70.70 & $73.51 \%$ & $77.14 \%$ & $88.84 \%$ \\
\hline SOFA & 0.83 & $0.30-3.33$ & 77.36 & 76.74 & $76.87 \%$ & $74.19 \%$ & $87.34 \%$ \\
\hline \multicolumn{8}{|c|}{ Validation cohort } \\
\hline Nomogram & 0.83 & $0.25-3.09$ & 82.00 & 73.45 & $75.00 \%$ & $66.67 \%$ & $87.15 \%$ \\
\hline APACHE ॥ & 0.82 & $0.38-3.37$ & 70.00 & 79.20 & $77.54 \%$ & $60.00 \%$ & $86.06 \%$ \\
\hline SOFA & 0.83 & $0.30-2.99$ & 78.00 & 73.89 & $74.64 \%$ & $70.83 \%$ & $86.90 \%$ \\
\hline
\end{tabular}

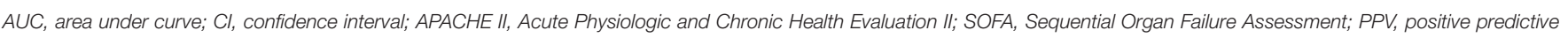
value; NPV, negative predictive value.

AKI as an independent risk factor using the LASSO Cox regression model.

Risk factors such as old age, malignant disease, and preexisting medical comorbidities may also attribute to the patient's underlying condition. Ana et al. (6) reported that elderly patients with intra-abdominal infection tend have a narrow therapeutic window, and old age is associated with significantly increased morbidity and mortality compared with younger patients. However, age was not identified in this study, which may be owing to the minimal differences between ages for all the patients had an advanced age [62.9 (61.5-64.3)] (Table 1). It is also indicated that cIAI mainly occurred in elderly patients. Interestingly, our study first identified sex as a risk factor, and male patients tended to have higher mortality and prolonged hospital stay. Similar observations were made by others in infectious diseases or septic shock, wherein males of any age showed worse prognosis (27). Females have better prognosis, and there is a hypotheses that it is probably ascribed to a higher neutrophilic inflammation and lower extracellular milieu's $\mathrm{pH}$ (28). Considering the comorbidities and malignant diseases, we chose Charlson score instead. Previous studies showed that Charlson score was significantly associated with all-cause mortality in patients with bacteremia (29) or sepsis (30), and our results corroborate these findings.

Achieving a prompt source control over the infection is crucial for abdominal infection management (4). Multi-pathogen infections often cause the failure of source control but are easily overlooked (31). This study identified infection with rare bacterium infection as the risk factor for cIAI prognosis. The underlying mechanism may be a lack of prompt and efficient antibacterial treatment when uncommon bacterium infection occurred. An emergent source control is necessary for cIAI with sepsis according to the most recent Surviving Sepsis Campaign Guidelines $(32,33)$. Antibiotic treatment mainly depends on experience, and cephalosporins and imipenem are commonly prescribed for cIAI (34). Furthermore, the infection rate of MDR bacteria was as high as $51.1 \%$ in this study, which was alarming.

The World Society of Emergency Surgery cIAIs Score Study (WISS) specifically constructed a WISS score, which includes severe sepsis or septic shock, healthcare-associated infections, delay in source control, origin of the IAIs, age, and immunosuppression to evaluate the severity of illness for patients with cIAI (35); it lacks verification and was principally used in surgical research since it was constructed in 2015 (36). Our newly constructed nomogram not only evaluated the whole condition (Charlson score and APACHE II score) but also included specific organ function (AGI, AKI) of patients with cIAI. Moreover, it emphasized the important role of timely specific pathogen identification for improving prognosis. The nomogram is more suitable for the evaluation of prognosis of cIAI in ICU. We even certificated the nomogram with a high calibration (Figures 4 A,B) and discriminative ability (Figures 5C,D).

This study has a few limitations mainly related to its retrospective design. Firstly, the evaluation of infection control effect was not generally conducted in this study, which is different from the WISS score. However, as far as we know, infection control was more like a result after we evaluated the illness and took measures. Further studies will evaluate the factors influencing infection control effect. Secondly, owing to the limitation of data collection, we have not identified drug resistance of each pathogen but simply designated pathogens as MDR. It will affect the result of our evaluation; further study may identify more risk factors. Thirdly, the practicality of the nomogram was potentially limited due to the lack of external validation. Therefore, additional validation using datasets from other countries are encouraged.

\section{CONCLUSIONS}

Our newly constructed nomogram, which included sex, AGI, AKI, rare bacterium infection, Charlson score, and APACHE II score, takes full consideration of the illness of cIAI, can predict its OS time accurately, and is considered a useful tool for risk stratification in CIAI.

\section{DATA AVAILABILITY STATEMENT}

The original contributions presented in the study are included in the article/Supplementary Material, further inquiries can be directed to the corresponding author/s. 


\section{ETHICS STATEMENT}

The studies involving human participants were reviewed and approved by Ruijin Hospital North. Written informed consent for participation was not required for this study in accordance with the national legislation and the institutional requirements.

\section{AUTHOR CONTRIBUTIONS}

All the authors have participated in clinical practice, literature retrieval, and viewpoint discussion in this article. SH and LC contributed to the writing of this article. SZ processed and analyzed the data. DC and JL revised this article. All authors read and approved the final manuscript.

\section{REFERENCES}

1. Vincent JL, Rello J, Marshall J, Silva E, Anzueto A, Martin CD, et al. International study of the prevalence and outcomes of infection in intensive care units. JAMA. (2009) 302:2323-9. doi: 10.1001/jama.2009.1754

2. Pelletier SJ, Raymond DP, Crabtree TD, Gleason TG, Pruett TL, Sawyer RG. Outcome analysis of intraabdominal infection with resistant gram-positive organisms. Surg Infect. (2002) 3:11-9. doi: 10.1089/109629602753681113

3. Schneider CP, Seyboth C, Vilsmaier M, Kuchenhoff H, Hofner B, Jauch KW, et al. Prognostic factors in critically ill patients suffering from secondary peritonitis: a retrospective, observational, survival time analysis. World J Surg. (2009) 33:34-43. doi: 10.1007/s00268-008-9805-4

4. van de Groep K, Verhoeff TL, Verboom DM, Bos LD, Schultz MJ, Bonten MJM, et al. Epidemiology and outcomes of source control procedures in critically ill patients with intra-abdominal infection. J Crit Care. (2019) 52:258-64. doi: 10.1016/j.jcrc.2019.02.029

5. De Waele J, Lipman J, Sakr Y, Marshall JC, Vanhems P, Barrera Groba $\mathrm{C}$, et al. Abdominal infections in the intensive care unit: characteristics, treatment and determinants of outcome. BMC Infect Dis. (2014) 14:420. doi: 10.1186/1471-2334-14-420

6. Berlin A, Johanning JM. Intraabdominal Infections in older adults. Clin Geriatr Med. (2016) 32:493-507. doi: 10.1016/j.cger.2016.02.002

7. Labricciosa FM, Sartelli M, Abbo LM, Barbadoro P, Ansaloni L, Coccolini F, et al., Epidemiology and risk factors for isolation of multi-drug-resistant organisms in patients with complicated intra-abdominal infections. Surg Infect. (2018) 19:264-72. doi: 10.1089/sur.2017.217

8. Friedrich AK, Cahan M. Intraabdominal infections in the intensive care unit. J Intensive Care Med. (2014) 29:247-54. doi: 10.1177/0885066613476465

9. Sartelli M, Catena F, Abu-Zidan FM, Ansaloni L, Biffl WL, Boermeester MA, et al. Management of intra-abdominal infections: recommendations by the WSES 2016 consensus conference. World J Emerg Surg. (2017) 12:22. doi: 10.1186/s13017-017-0132-7

10. van Ruler O, Kiewiet J, Boer KR, Lamme B, Gouma DJ, Boermeester MA, et al. Failure of available scoring systems to predict ongoing infection in patients with abdominal sepsis after their initial emergency laparotomy. BMC Surg. (2011) 11:38. doi: 10.1186/1471-2482-11-38

11. Blot S, De Waele JJ. Critical issues in the clinical management of complicated intra-abdominal infections. Drugs. (2005) 65:1611-20. doi: 10.2165/00003495-200565120-00002

12. Sartelli M, Catena F, Ansaloni L, Moore E, Malangoni M, Velmahos G, et al. Complicated intra-abdominal infections in a worldwide context: an observational prospective study (CIAOW study). World J. Emerg. Surg. (2013) 8:1. doi: 10.1186/1749-7922-8-1

13. Plaudis H, Rudzats A, Melberga L, Kazaka I, Suba O, Pupelis G. Abdominal negative-pressure therapy: a new method in countering abdominal compartment and peritonitis - prospective study and

\section{FUNDING}

This work was supported by the National Natural Science Foundation of China (Grant No. 81873944).

\section{ACKNOWLEDGMENTS}

We are indebted to all the intensivists who provided the clinical and demographic data of patients with complicated intraabdominal infection.

\section{SUPPLEMENTARY MATERIAL}

The Supplementary Material for this article can be found online at: https://www.frontiersin.org/articles/10.3389/fmed. 2021.627416/full\#supplementary-material

critical review of literature. Ann Intensive Care. (2012) 2(Suppl 1):S23. doi: 10.1186/2110-5820-2-S1-S23

14. Suarez-de-la-Rica A, Maseda E, Anillo V, Tamayo E, García-Bernedo CA, Ramasco F, et al. Biomarkers (procalcitonin, $\mathrm{C}$ reactive protein, and lactate) as predictors of mortality in surgical patients with complicated intra-abdominal infection. Surg. Infect. (2015) 16:346-51. doi: 10.1089/sur.2014.178

15. Suarez-de-la-Rica A, Anillo V, Montero A, Hernandez-Gancedo C, LopezTofiño A, Gilsanz F, et al. Risk factors for acute kidney injury in critically ill patients with complicated intra-abdominal infection. J Crit Care. (2017) 38:104-8. doi: 10.1016/j.jcrc.2016.10.031

16. Das K, Ozdogan M, Karateke F, Uzun AS, Sozen S, Ozdas S. Comparison of APACHE II, P-POSSUM and SAPS II scoring systems in patients underwent planned laparotomies due to secondary peritonitis. Ann Ital Chir. (2014) 85:16-21.

17. Augustin P, Tran-Dinh A, Valin N, Desmard M, Crevecoeur MA, MullerSerieys C, et al. Pseudomonas aeruginosa post-operative peritonitis: clinical features, risk factors, and prognosis. Surg Infect. (2013) 14:297-303. doi: 10.1089/sur.2012.084

18. Kulkarni SV, Naik AS, Subramanian N Jr. APACHE-II scoring system in perforative peritonitis. Am J Surg. (2007) 194:549-52. doi: 10.1016/j.amjsurg.2007.01.031

19. Chong YP, Bae IG, Lee SR, Chung JW, Jun JB, Choo EJ, et al. Clinical and economic consequences of failure of initial antibiotic therapy for patients with community-onset complicated intra-abdominal infections. PLoS ONE. (2015) 10:e0119956. doi: 10.1371/journal.pone.0119956

20. Reintam Blaser A, Malbrain ML, Starkopf J, Fruhwald S, Jakob SM, De Waele $\mathrm{J}$, et al. Gastrointestinal function in intensive care patients: terminology, definitions and management. Recommendations of the ESICM working group on abdominal problems. Intensive Care Med. (2012) 38:384-94. doi: 10.1007/s00134-011-2459-y

21. Zhang D, Li H, Li Y, Qu L. Gut rest strategy and trophic feeding in the acute phase of critical illness with acute gastrointestinal injury. Nutr Res Rev. (2019) 32:176-82. doi: 10.1017/S0954422419000027

22. Xing J, Zhang Z, Ke L, Zhou J, Qin B, Liang H, et al. Enteral nutrition feeding in Chinese intensive care units: a cross-sectional study involving 116 hospitals. Crit Care. (2018) 22:229. doi: 10.1186/s13054-018-2159-x

23. Li H, Zhang D, Wang Y, Zhao S. Association between acute gastrointestina injury grading system and disease severity and prognosis in critically ill patients: a multicenter, prospective, observational study in China. J Crit Care. (2016) 36:24-8. doi: 10.1016/j.jcrc.2016.05.001

24. Hu B, Sun R, Wu A, Ni Y, Liu J, Guo F, et al. Severity of acute gastrointestinal injury grade is a predictor of all-cause mortality in critically ill patients: a multicenter, prospective, observational study. Crit Care. (2017) 21:188. doi: 10.1186/s13054-017-1780-4

25. Ding L, Chen HY, Wang JY, Xiong HF, He WH, Xia L, et al. Severity of acute gastrointestinal injury grade is a good predictor of mortality in critically 
ill patients with acute pancreatitis. World J Gastroenterol. (2020) 26:514-23. doi: 10.3748/wjg.v26.i5.514

26. Uchino S, Kellum JA, Bellomo R, Doig GS, Morimatsu H, Morgera S, et al. Acute renal failure in critically ill patients: a multinational, multicenter study. JAMA. (2005) 294:813-8. doi: 10.1001/jama.294.7.813

27. Papathanassoglou E, Middleton N, Benbenishty J, Williams G, Christofi MD, Hegadoren K. Systematic review of gender-dependent outcomes in sepsis. Nurs Crit Care. (2017) 22:284-92. doi: 10.1111/nicc.12280

28. Casimir GJ, Lefevre N, Corazza F, Duchateau J, Chamekh M. The acid-base balance and gender in inflammation: a mini-review. Front Immunol. (2018) 9:475. doi: 10.3389/fimmu.2018.00475

29. Eskesen AN, Belle MA, Blomfeldt A. Predictors of one-year all-cause mortality and infection-related mortality in patients with Staphylococcus aureus bacteraemia. Infect Dis. (2018) 50:743-8. doi: 10.1080/23744235.2018.14 70666

30. Oh TK, Jeon YT, Do SH, Hwang JW. Pre-operative assessment of 30-day mortality risk after major surgery: the role of the quick sequential organ failure assessment: a retrospective observational study. Eur J Anaesthesiol. (2019) 36:688-94. doi: 10.1097/EJA.00000000000 00957

31. Tellor B, Skrupky LP, Symons W, High E, Micek ST, Mazuski JE. Inadequate source control and inappropriate antibiotics are key determinants of mortality in patients with intra-abdominal sepsis and associated bacteremia. Surg Infect. (2015) 16:785-93. doi: 10.1089/sur.2014.166

32. Levy MM, Evans LE, Rhodes A. The surviving sepsis campaign bundle: 2018 update. Intensive Care Med. (2018) 44:925-8. doi: 10.1007/s00134-018-5085-0
33. Singer M, Deutschman CS, Seymour CW, Shankar-Hari M, Annane D, Bauer $\mathrm{M}$, et al. The third international consensus definitions for sepsis and septic shock (sepsis-3). JAMA. (2016) 315:801-10. doi: 10.1001/jama.2016.0287

34. Liu L, Ni Y. Antimicrobial susceptibilities of specific syndromes created with organ-specific weighted incidence antibiograms (OSWIA) in patients with intra-abdominal infections. BMC Infect Dis. (2018) 18:584. doi: 10.1186/s12879-018-3494-x

35. Sartelli M, Abu-Zidan FM, Catena F, Griffiths EA, Di Saverio S, Coimbra $\mathrm{R}$, et al. Global validation of the WSES sepsis severity Score for patients with complicated intra-abdominal infections: a prospective multicentre study (WISS study). World J Emerg Surg. (2015) 10:61.

36. Abdel-Kader S, Sartelli M, Abu-Zidan FM. Complicated intra-abdominal infections: a prospective validation study of the WSES sepsis severity score. Singapore Med J. (2019) 60:317-21. doi: 10.11622/smedj.2018120

Conflict of Interest: The authors declare that the research was conducted in the absence of any commercial or financial relationships that could be construed as a potential conflict of interest.

Copyright (C) 2021 Huang, Chen, Liu, Zhang, Zhang, Wen, Chen and Chen. This is an open-access article distributed under the terms of the Creative Commons Attribution License (CC BY). The use, distribution or reproduction in other forums is permitted, provided the original author(s) and the copyright owner(s) are credited and that the original publication in this journal is cited, in accordance with accepted academic practice. No use, distribution or reproduction is permitted which does not comply with these terms. 